\title{
Renal Effect of Lopinavir/Ritonavir and Sulfamethoxazole/Trimethoprim in Albino Rats
}

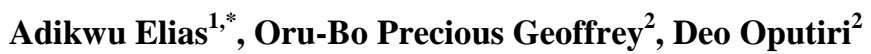 \\ ${ }^{1}$ Department of Pharmacology, Faculty of Basic Medical Sciences, College of Health Sciences, University of Port Harcourt, Choba, \\ Rivers State, Nigeria \\ ${ }^{2}$ Department of Pharm Tech. College of Health Technology, Otuogidi, Ogbia, L. G. A., Bayelsa State \\ *Corresponding author: adikwuelias@gmail.com
}

Received September 01, 2014; Revised September 12, 2014; Accepted September 17, 2014

\begin{abstract}
Lopinavir/ritonavir and sulfamethoxazole/ trimethoprim have been individually associated with renal adverse events which could be characterized by electrolyte imbalance. The concurrent use of these drugs in the management of HIV and co infections may impair renal function; therefore this work evaluates the renal effect of the co administration of these drugs in rats. Seventy five (75) animals which were divided into five (5) groups were used in this study. Group A which served as control contained fifteen (15) animals which were treated with 1\% ethanol orally. Group B-E which contained fifteen (15) animals each was further subdivided into three (3) subgroups of five (5) animals each. Animals in these groups were treated with oral doses of SMX/TMP (11.2/2.3mg/kg), LPV/r $(11.4 / 2.9 \mathrm{mg} / \mathrm{kg})$ and combined doses of SMX/TMP + LPV/r for 2-8 weeks respectively. Serum levels of potassium, sodium and chloride were evaluated. Kidney tissues were evaluated for catalase (CAT), glutathione peroxidase $\left(\mathrm{GSHP}_{\mathrm{X}}\right)$ and histopathological changes. Results showed that treatment with LPV/r, SMX/TMP and co administered SMX/TMP + LPV/r had no significant time dependent effect on serum potassium, sodium and chloride. Treatment with SMX/TMP produced time dependent decrease in kidney CAT and GSHP x $_{\mathrm{X}}$ while LPV/r had no significant effect on kidney CAT and GSHP ${ }_{\mathrm{x}}$. No significant synergistic effects were observed on CAT and GSHP $\mathrm{P}_{\mathrm{X}}$ level when these agents were co administered. Single and combined doses of these agents did not produce morphological changes in the kidney of treated animals. Conclusion: Concurrent use of LPV/r and SMX/TMP in the management of a HIV and co infection may not have deleterious effect on serum electrolytes and kidney structure.
\end{abstract}

Keywords: toxicity, renal, protease Inhibitors, cotrimoxazole, rats

Cite This Article: Adikwu Elias, Oru-Bo Precious Geoffrey, and Deo Oputiri, "Renal Effect of Lopinavir/Ritonavir and Sulfamethoxazole/Trimethoprim in Albino Rats." American Journal of Pharmacological Sciences, vol. 2, no. 5 (2014): 77-81. doi: 10.12691/ajps-2-5-1.

\section{Introduction}

Protease inhibitors boosted with ritonavir are components of highly active antiretroviral therapy use in the management of naive and experienced HIV-infected patients. [1] Protease inhibitors are commonly used options for salvage therapy in HIV management and one of the frequently used options is the lopinavir /ritonavir (LPV/r) combination. The pharmacokinetic benefits of lopinavir/ritonavir combination include increase plasma concentration since both are substrates for and inhibitors of cytochrome P4503A [2].

Sulfamethoxazole/ trimethoprim (SMX/TMP) is a fixed dose combination that provides a broad coverage against bacteria, protozoa and fungi. WHO recommends SMX/TMP as prophylaxis in all adults with WHO clinical stage 2, 3 or 4 HIV infection especially in resource limited areas. In vicinities with high prevalence of HIV infection it is stipulated that all infected adults be treated irrespective of disease stage or CD4 cell count. [3] Clinical trials have reported decreased mortality and morbidity in adults with
HIV on SMX/TMP prophylaxis. [4] Sulfamethoxazole/ trimethoprim and antiretroviral regimens containing $\mathrm{LPV} / \mathrm{r}$ are concurrently used in the management of HIV and co infections. [5] LPV/r and SMX/TMP have been individually implicated in renal adverse events with electrolyte imbalance [6] hence the concurrent use of these drugs may add more toxicological burden on the kidney.

The kidney is an essential organ which is associated with several excretory and regulatory functions including electrolyte balance. The passage of drugs and chemical substances through the kidney could induce renal damage which may impair its excretory and regulatory functions. $[7,8]$ Drugs and their metabolites could be actively concentrated in the renal cortex and proximal tubular cells which may induce oxidative stress leading to decrease kidney antioxidants capacity. This condition may precipitate architectural kidney damage leading to decrease in kidney functions which include electrolyte balance. $[9,10]$ Due to deleterious effects of some drugs on the kidney and the association of LPV/r and SMX/TMP with individual adverse renal events, the concurrent use of these drugs may impair renal function. This work evaluates the toxicological effects of the co administration of these 
agents on serum electrolytes, kidney antioxidant enzymes and architecture of albino rats.

\section{Materials and Methods}

\subsection{Drugs}

Lopinavir/ritonavir (LPV/r) used in this work was manufactured by Myland Laboratories Limited India. Other ingredients in the LPV/r tablet include colloidal silicon dioxide, copovidone, sodium stearyl fumarate and sorbitan monolaurate. Sulfamethoxazole/Trimethoprim (SMX/TMP) used in this study was manufactured by CSPC Ouyi Pharmaceuticals China. Other ingredients in SMX/TMP tablets include docusate sodium, magnesium stearate, maize starch, silica and sodium lauryl sulphate.

\subsection{Animals}

The animals used in this research work were obtained from the animal house of the Department of Pharmacology and Toxicology, Madonna University, Elele, Rivers State. The animals were allowed free access to food and water ad libitum and were allowed to acclimatize for 14 days. Animals were handled according to Helsinki declaration on the handling and use of animals.

\subsection{Dose Selection}

$11.2 / 2.3 \mathrm{mg} / \mathrm{kg}$ of SMX-TMP and $11.4 / 2.9 \mathrm{mg} / \mathrm{kg}$ of $\mathrm{LPV} / \mathrm{r}$ were used in this study. Doses used are within the clinically recommended dose range [11].

\subsection{Preparation of Drug}

Lopinavir/ritonavir tablets were crushed and dissolved in $1 \%$ ethanol while Sulphamethoxazole/ trimethoprim tablets were also crushed and dissolved in sterile water [12].

\subsection{Grouping of Animals}

Seventy five healthy male rats were weighed and housed in a large mesh cage. The rats were divided into five groups A B C D and E.

\subsection{Drug Administration}

Group A: This group served as the control and contained fifteen animals which were treated with1\% ethanol orally throughout the duration of study.

Group B: This group contained 15 animals which were further divided into three subgroups (B1-B3). Animals in sub group B1 were treated with $11.2 / 2.3 \mathrm{mg} / \mathrm{kg}$ of SMX/TMP. Animals in subgroup B2 were treated with $11.4 / 2.9 \mathrm{mg} / \mathrm{kg}$ of LPV/r. Animals in subgroup B3 were treated with combined doses of SMX/TMP + LPV/r. All animals in this group were treated for 2 weeks.

Group C: This group contained 15 animals which were further divided into three subgroups (C1-C3). Animals in sub group C1 were treated with $11.2 / 2.3 \mathrm{mg} / \mathrm{kg}$ of SMX/TMP. Animals in subgroup C2 were treated with $11.4 / 2.9 \mathrm{mg} / \mathrm{kg}$ of LPV/r. Animals in C3 were treated with combined doses of SMX/TMP + LPV/r. All animals in this group were treated for 4 weeks.
Group D: This group contained 15 rats which were further divided into three subgroups (D1-D3). Animals in sub group D1 were treated with $11.2 / 2.3 \mathrm{mg} / \mathrm{kg}$ of SMX/TMP. Animals in subgroup D2 were treated with $11.4 / 2.9 \mathrm{mg} / \mathrm{kg}$ of LPV/r. Animals in D3 were treated with combined doses of SMX/TMP + LPV/r. All animals in this group were treated for 6 weeks.

Group E: This group contained 15 animals which were further divided into three subgroups (E1-E3). Animals in sub group E1 were treated with $11.2 / 2.3 \mathrm{mg} / \mathrm{kg}$ of SMX/TMP. Animals in subgroup E2 were treated with $11.4 / 2.9 \mathrm{mg} / \mathrm{kg}$ of LPV/r. Animals in E3 were treated with combined doses of SMX/TMP + LPV/r. All animals in this group were treated for 8 weeks.

\subsection{Collection of Sample for Analysis}

Animals were sacrificed using chloroform anesthesia at the end of 2, 4, 6 and 8 weeks of treatment respectively. Blood sample was collected from the common carotid artery. The sample was allowed to clot and centrifuged at 1000 rpm for 5mins using Uniscope centrifuge and serum separated for analysis. Rats were dissected kidney was collected and analyzed for histopathological changes.

\subsection{Preparation of Tissue Homogenate}

The kidney tissues were homogenized using $0.1 \%$ Triton X-100 buffer ( $\mathrm{pH} 7.4)$. The homogenate was centrifuged at $12,000 \mathrm{rpm} \&$ at $4{ }^{\circ} \mathrm{C}$ for $30 \mathrm{~min}$ and the supernatant was used as sample for biochemical investigations [13].

\subsection{Evaluation of Kidney Catalase and Glutathione}

Glutathione Peroxidase (GSHPx) activity was evaluated as reported by Wendel, 1980 [14] while the activity of Catalase (CAT) was assayed by the method of Aebi, 1984 [15].

\subsection{Evaluation of Serum Electrolytes}

Potassium and sodium were determined using flame photometric methods, while chloride levels were determined using titrimetric methods [16].

\subsection{Histopathological Evaluation}

For light microscopic examination, kidney tissues from each group were fixed with $10 \%$ buffered formalin. The specimens were dehydrated in ascending grades of ethanol, cleared in xylene and embedded in paraffin wax. Sections of $5 \mathrm{~cm}$ in thickness were prepared and stained with Haematoxylin and Eosin [17] and then examined under light microscopy. The photomicrographs of the relevant stained sections were taken with the aid of a light microscope.

\subsection{Statistical Analysis}

Results were expressed as mean \pm S.E.M. Statistical analysis was done with the aid of SPSS for windows; SPSS Inc., Chicago, Standard version 14.0 to determine difference between mean using One Way Analysis of Variance (ANOVA). 


\section{Results}

\subsection{Effect on Serum Electrolytes}

In this study treatment with SMX/TMP for 2-8 weeks did not produced any significant change in serum potassium level $(\mathrm{P}>0.05)$ with respect to the control. No significant $(\mathrm{P}>0.05)$ change in serum potassium level was noted in animals treated with LPV/r for 2-8 weeks with respect to the control. Combined treatment with $\mathrm{SMX} / \mathrm{TMP}+\mathrm{LPV} / \mathrm{r}$ did not produce any significant ( $P>0.05)$ change in serum potassium level after treatment for 2-8 weeks when compared with the control [Table 1].

Table 1. Effect of lopinavir/ritonavir, sulfamethoxazole/trimethoprim and their combination on serum potassium (mmol/l) of albino rats

\begin{tabular}{lcccc}
\hline Dose mg/kg & Week2 & Week4 & Week 6 & Week 8 \\
CONTROL & $4.35 \pm 0.8$ & $4.45 \pm 2.7$ & $4.65 \pm 1.8$ & $4.55 \pm 3.6$ \\
SMX/TMP (11.2/2.3) & $4.43 \pm 3.2$ & $4.67 \pm 0.3$ & $4.70 \pm 3.4$ & $5.85 \pm 5.3$ \\
LPV/r & $4.50 \pm 2.4$ & $4.83 \pm 4.3$ & $4.95 \pm 2.5$ & $5.35 \pm 1.3$ \\
$\mathbf{( 1 1 . 4 / 2 . 9 )}$ & $4.73 \pm 4.2$ & $4.91 \pm 1.1$ & $5.13 \pm 3.5$ & $5.58 \pm 2.8$ \\
SMX/TMP+LPV/r &
\end{tabular}

Results are express as mean \pm SEM, the superscript $(*)$ means significan difference with respect to the control at $\mathrm{p}<0.05$ (ANOVA).

Sulfamethoxazole/ trimethoprim did not produce any significant $(\mathrm{P}>0.05)$ change in serum sodium level after treatment for 2-8 weeks when compared with the control. Exposure of animals to LPV/r for 2-8 weeks had no significant change in serum sodium levels with respect to the control. Treatment with combined doses of SMX/TMP $+\mathrm{LPV} / \mathrm{r}$ had no significant $(\mathrm{P}>0.05)$ effect on serum sodium level after treatment for 2-8 weeks with respect to the control. [Table 2] There was no significant $(\mathrm{P}>0.05)$ change in chloride level observed in animals treated with individual doses of SMX/TMP and LPV/r for 2-8 weeks respectively when compared with the control. Also concurrent treatment with SMX/TMP + LPV/r for 2-8 weeks did not produce any significant $(\mathrm{P}>0.05)$ change in serum chloride level with respect to the control [Table 3].

Table 2. Effect of lopinavir/ritonavir, sulfamethoxazole/trimethoprim and their combination on serum sodium (mmol/l) of male albino rats

\begin{tabular}{lcccc}
\hline Dose mg/kg & Week2 & Week4 & Week 6 & Week 8 \\
CONTROL & $120.5 \pm 4.3$ & $121.7 \pm 1.5$ & $123.2 \pm 2.2$ & $125.3 \pm 3.1$ \\
SMX/TMP (11.2/2.3) & $121.3 \pm 4.9$ & $120.5 \pm 1.6$ & $121.3 \pm 0.4$ & $122.2 \pm 2.1$ \\
$\mathbf{L P V / r}$ & $120.7 \pm 2.8$ & $122.4 \pm 0.6$ & $121.6 \pm 4.7$ & $121.0 \pm 7.0$ \\
$\mathbf{( 1 1 . 4 / 2 . 9 )}$ & $121.2 \pm 0.7$ & $122.7 \pm 2.8$ & $121.0 \pm 5.1$ & $120.5 \pm 2.5$ \\
SMX/TMP+LPV/r & &
\end{tabular}

Results are express as mean \pm SEM, the superscript $\left(^{*}\right)$ means significant difference with respect to the control at $\mathrm{p}<0.05$ (ANOVA).

Table 3. Effect of lopinavir/ritonavir, sulfamethoxazole/trimethoprim and their combination on serum chloride ( $\mathrm{mmol} / \mathrm{l})$ of albino rats

\begin{tabular}{lcccc}
\hline Dose mg/kg & Week2 & Week4 & Week 6 & Week 8 \\
CONTROL & $103.3 \pm 1.1$ & $105.5 \pm 2.0$ & $103.3 \pm 3.1$ & $104.2 \pm 4.1$ \\
SMX/TMP (11.2/2.3) & $103.5 \pm 0.6$ & $103.3 \pm 4.6$ & $105.2 \pm 2.3$ & $104 \pm 3.7$ \\
LPV/r & $104.5 \pm 4.3$ & $104.9 \pm 2.7$ & $106.7 \pm 1.1$ & $105.3 \pm 2.6$ \\
$\mathbf{( 1 1 . 4 / 2 . 9 )}$ & $104.7 \pm 4.0$ & $103.3 \pm 1.0$ & $105.2 \pm 2.53$ & $105.8 \pm 1.9$ \\
SMX/TMP+LPV/r &
\end{tabular}

Results are express as mean \pm SEM, the superscript $(*)$ means significant difference with respect to the control at $\mathrm{p}<0.05$ (ANOVA).

\subsection{Effect on Kidney Catalase, Glutathione Peroxidase and Kidney Histopathology}

Treatment with LPV/r for 2-8 weeks produced an insignificant $(\mathrm{P}>0.05)$ decrease in kidney CAT and $\mathrm{GSHP}_{\mathrm{X}}$ with respect to the control. CAT and $\mathrm{GSHP}_{\mathrm{X}}$ decreased time dependently in animals exposed to SMX/TMP with significance $(\mathrm{P}<0.05)$ in week 8 when compared with the control. Treatment with combined doses of these agents produced significant $(\mathrm{P}<0.05)$ decrease in CAT and $\mathrm{GSHP}_{\mathrm{X}}$ in week 8 with respect to the control. [Table 4 and Table 5] There were no histopathological changes observed in kidney architecture of rats treated for 2 weeks with SMX/TMP, LPV/r and combined doses of $\mathrm{SMX} / \mathrm{TMP}+\mathrm{LPV} / \mathrm{r}$ with respect to the control [Figure 1, Figure 2, Figure 3 and Figure 4].

Table 4. Effect of lopinavir/ritonavir, sulfamethoxazole/trimethoprim and their combination on kidney CAT (u/mg protein) of albino rats

$\begin{array}{lcccc}\text { Dose mg/kg } & \text { Week2 } & \text { Week4 } & \text { Week 6 } & \text { Week 8 } \\ \text { Control } & 30.5 \pm 3.1 & 31.2 \pm 5.2 & 31.1 \pm 2.0 & 32.4 \pm 1.3 \\ \begin{array}{l}\text { SMX/TMP } \\ (\mathbf{1 1 . 2 / 2 . 3 )}\end{array} & 30.1 \pm 4.5 & 29.3 \pm 2.4 & 27.6 \pm 2.3 & 18.1 \pm 2.4^{*} \\ \mathbf{L P V / r} & 29.8 \pm 5.4 & 28.6 \pm 3.4 & 29.1 \pm 4.1 & 28.7 \pm 3.0 \\ \mathbf{( 1 1 . 4 / 2 . 9 )} & 29.1 \pm 3.1 & 28.2 \pm 1.5 & 27.9 \pm 2.7 & 18.1 \pm 1.8^{*} \\ \text { SMX/TMP+LPV/r } & 27.0\end{array}$

Results are express as mean \pm SEM, the superscript $(*)$ means significant difference with respect to the control at $\mathrm{p}<0.05$ (ANOVA).

Table 5. Effect of lopinavir/ritonavir, sulfamethoxazole/trimethoprim and combination on kidney GSH $P_{X}$ (u/mg protein) of albino rats

\begin{tabular}{lcccc}
\hline Dose mg/kg & Week2 & Week4 & Week 6 & Week 8 \\
CONTROL & $22.4 \pm 3.1$ & $22.7 \pm 2.5$ & $23.1 \pm 2.0$ & $24.2 \pm 3.2$ \\
$\begin{array}{l}\text { SMX/TMP } \\
(\mathbf{1 1 . 2} / \mathbf{2 . 3 )}\end{array}$ & $22.1 \pm 4.5$ & $21.9 \pm 2.4$ & $18.6 \pm 3.5$ & $13.1 \pm 2.4^{*}$ \\
$\mathbf{L P V} / \mathbf{r}$ & $21.3 \pm 4.1$ & $20.7 \pm 3.2$ & $19.8 \pm 2.3$ & $19.5 \pm 3.0$ \\
$\mathbf{( 1 1 . 4 / 2 . 9 )}$ & & & & \\
SMX/TMP+LPV/r & $21.3 \pm 3.2$ & $20.5 \pm 2.1$ & $18.0 \pm 2.5$ & $12.1 \pm 2.8^{*}$ \\
\hline
\end{tabular}

Results are express as mean \pm SEM, the superscript $(*)$ means significant difference with respect to the control at $\mathrm{p}<0.05$ (ANOVA).

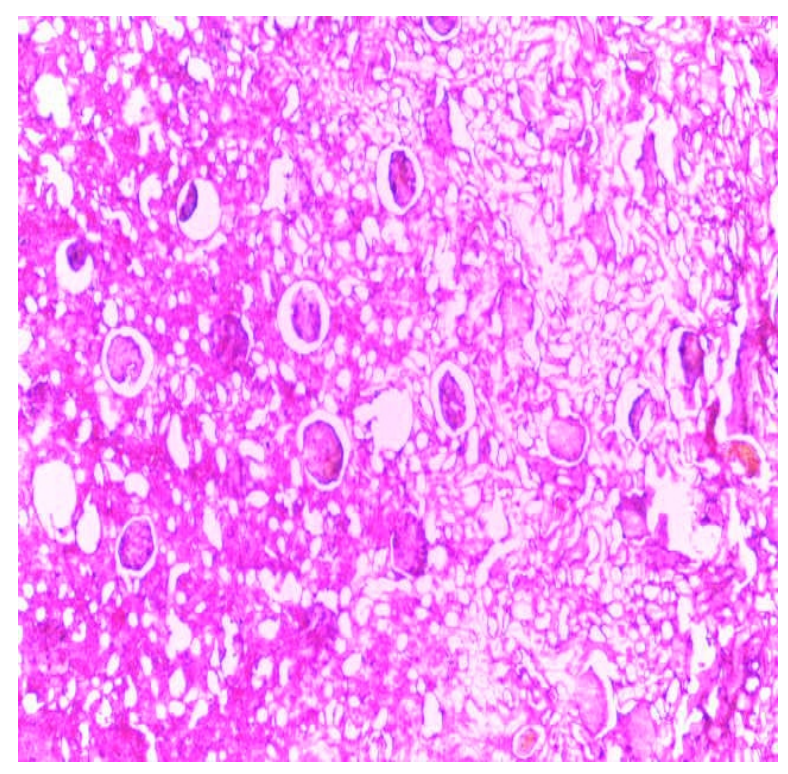

Figure 1. This is the photomicrograph of the H\&E section of the kidney of control animal treated with $1 \%$ ethanol showing normal glomeruli, tubules and interstitium. Mag x400 


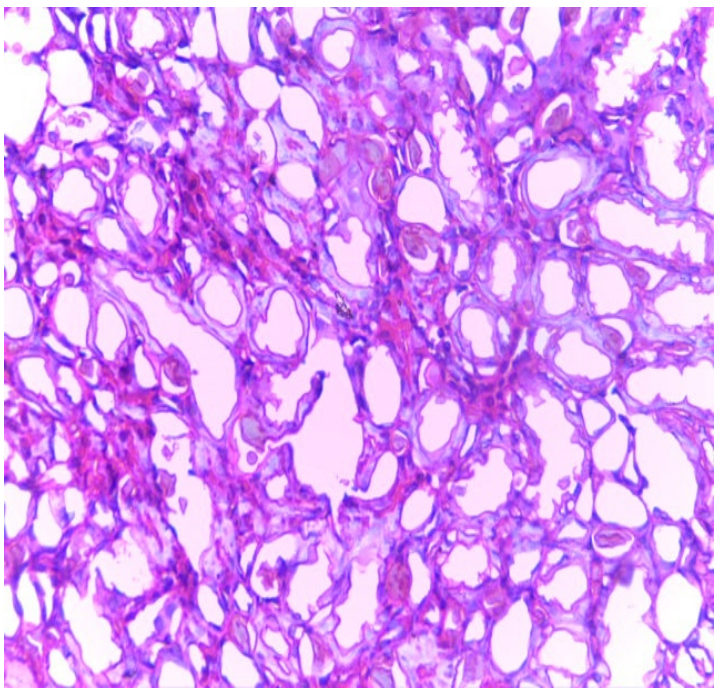

Figure 2. This is the photomicrograph of the H\&E section of the kidney of animals treated with $\mathrm{LPV} / \mathrm{r}(11.4 / 2.3 \mathrm{mg} / \mathrm{kg})$ for 2 weeks. It shows normal glomeruli in number and structure. Mag x400

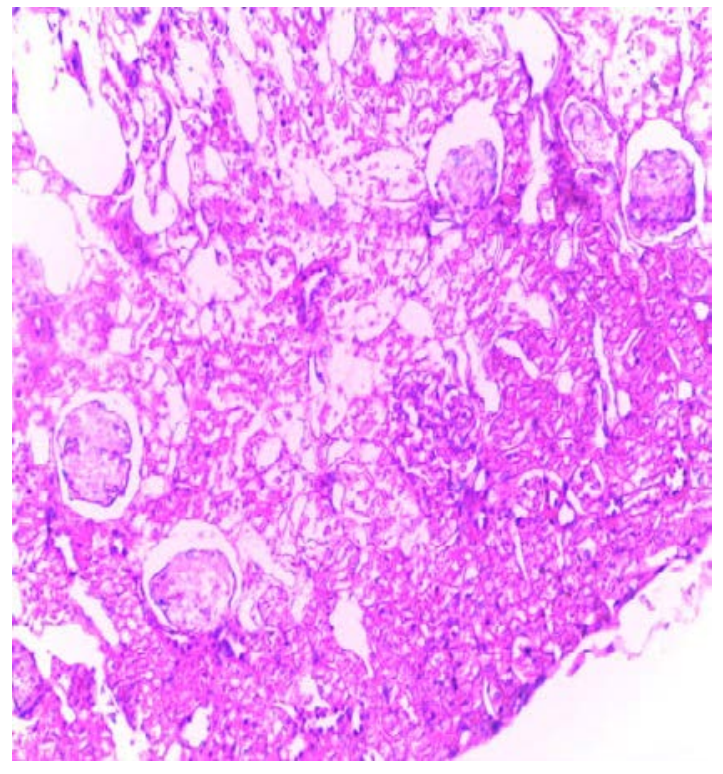

Figure 3. This is the photomicrograph of the H\&E section of the kidney of animals treated with SMX/TMP $(11.4 / 2.9 \mathrm{mg} / \mathrm{kg})$ for 2 weeks. It shows normal glomeruli in number and structure. Mag x400

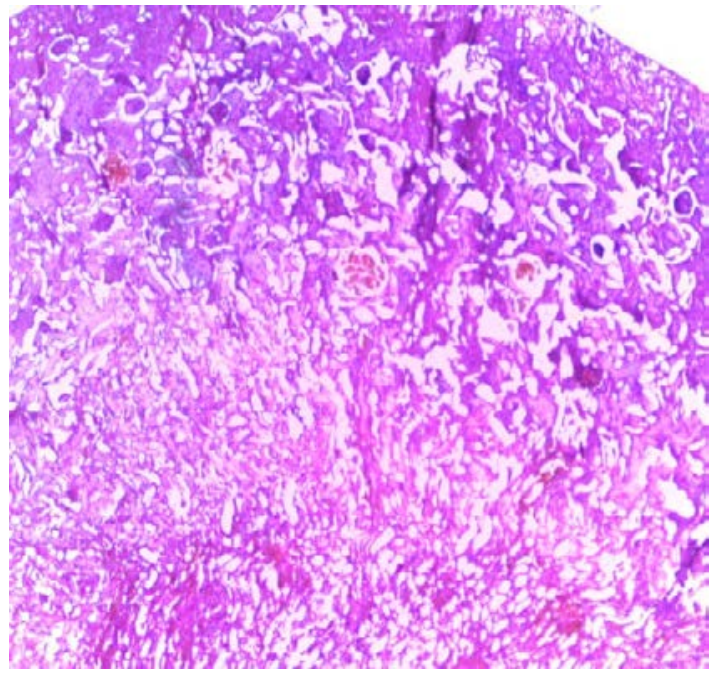

Figure 4. This is the photomicrograph of the H\&E section of the kidney of animals treated with SMX/TMP +LPV/r for 2weeks. It shows normal glomeruli in number and structure. Mag x400

\section{Discussion}

Electrolytes excretion is regulated by the kidney, fluctuations in their levels could trigger cardiac adverse events and other complications; hence routine evaluation of serum electrolytes during drug therapy is of clinical importance. [18] In this study treatment with SMX/TMP had no effect on potassium level which disagrees with some reports [19]. This difference in report could be associated with variation in dose because most reported cases of SMX/TMP induced hyperkalemia were associated with higher doses. [20] Some authors who reported SMX/TMP induced hyperkalemia associated it with its trimethoprim component which is a heterocyclic weak base and is structurally related to potassium- sparing diuretics like amiloride and triamterene. Trimethoprim acts as a potassium- sparing diuretic by blocking the amiloride- sensitive sodium channels in the distal tubule $[21,22]$.

Obirikorang and colleagues reported that treatment of HIV patients with antiretroviral drugs could be associated with fluctuations in potassium level [23] which is at variance with our observation in animals treated with $\mathrm{LPV} / \mathrm{r}$. Variation in observations could be tied to differences in species and duration of therapy as observed in their work. Due to observation in this work, concurrent use of SMX/TMP and LPV/r may not have deleterious effect on serum potassium level. Treatment with LPV/r had no effect on sodium level which is in resonance with the work of obirikorang et al., 2014. [23]. Noto and coworkers reported a case of SMX/TMP induced hyponatremia in a HIV patient [24] which is inconsistent with our findings. Some researchers who reported SMX/TMP induced hyponatremia attributed it to the ability of SMX/TMP to inhibit sodium re absorption in the cortical collecting tubules leading to hypovolemia and secretion of antidiuretic hormone. [25] Concurrent use of SMX/TMP and LPV/r may not have any toxic effect on serum sodium level with respect to observation in this study.

Hyperchloremic non- anion gap metabolic acidosis was reported in an AIDS patient treated for pneumocystic carinii pneumonia with high dose intravenous SMX/TMP [26] which disagrees with findings in this work. This disagreement could be associated with differences in drug dose and route of administration. Treatment with LPV/r had no significance effect on chloride level and no synergistic effect was observed on serum sodium level when LPV/r was co administered with SMX/TMP. This shows that concurrent use of these agents may not be deleterious to serum sodium level.

Kidney contains glutathione peroxidase which participates in the reduction of peroxides with concomitant formation of oxidized glutathione disulfide preventing kidney injury. Decrease in kidney glutathione peroxidase $\left(G S H P_{X}\right)$ level observed in this study in animals treated with SMX/TMP is a marker of kidney insult which could be associated with oxidative stress. $[27,28]$ SMX/TMP associated decrease in GSHP $_{X}$ wasn't potentiated when co administered with $\mathrm{LPV} / \mathrm{r}$ which shows that at the dose level used in this study, concurrent use of these agents may not be associated with kidney injury. Decrease in kidney CAT level noted in animals treated with SMX/TMP is also a sign of kidney injury 
because CAT is one of the antioxidant enzymes that form the first line of defense against ROS and decrease in their activities connotes oxidative insult on tissues. [29] Concurrent use of SMX/TMP and LPV/r may be safe on kidney CAT due to observations in this work. Morphological changes in kidneys have been reported in animals treated with individual doses of SMX/TMP and $\mathrm{LPV} / \mathrm{r}$ respectively which disagrees with observations in this current work. [30,31] This could also be tied to variations in dose, route and duration of drug administration as observed in their studies.

\section{Conclusion}

Based on observations in this study the concurrent use of SMX/TMP and LPV/r in the management of HIV and co infections may not have deleterious effect of serum electrolytes and kidney structure.

\section{References}

[1] Palella FJ, Delaney KM, Moorman AC. Declining morbidity and mortality among patients with advanced human immunodeficiency virus infection. HIV outpatient study investigators. $N$ Engl J Med. 338: 853-60, 1998.

[2] Dicenzo, R. Luque, A, Larppanichpoonphol, P, and R. Reichman Association of total bilirubin with indinavir and lopinavir plasma concentrations in HIV-infected patients receiving three different double-boosted dosing regimens. Jour of Antimicrob Chemother. 58, 393-400, 2006.

[3] Suthar AB, Granich R, Merminc J \& Van RieaA. Effect of cotrimoxazole on mortality in HIV-infected adults on antiretroviral therapy: a systematic review and meta-analysis. Bull World Health Organ; 90: 128-138. 2012.

[4] Anglaret X, Chene G, Attia A, Toure S, Lafont S, Combe P. Early chemoprophylaxis with trimethoprim-sulphamethoxazole for HIV1-infected adults in Abidjan, Cote d'Ivoire: a randomised trial. Lancet, 353: 1463-8. 1999.

[5] A. Allroggen A, Frese A, Rahmann M, Gaubitz I.W, Evers. HS hiv associated arthritis: case report and review of the literature. Eur J Med Res. 10: 305-308 2005.

[6] Giraud O, Thomas F, Aussavy F, Jupas J, and Ravaud Y. Hyperkaliemia and acute renal failure after overdose of trimethoprim- sulfamethoxazole in one AIDS patient," Annales de Medecine Interne, 148 (2) 185-186. 1997.

[7] Perazella MA. Renal Vulnerability to Drug Toxicity. Clin. J. Am. Soc. Nephrol. 4:1275-1283. 2009.

[8] Ferguson MA and Waikar SS. Established and Emerging Markers of Renal Function. Clin. Chem. 58(4): 680-689. 2012.

[9] Singh,N.P, Ganguli A, and Prakash A, Drug-induced Kidney Diseases.2003 JAPI. 51. 971-979.

[10] Abdel-Moneim A.M and Said K M. Acute Effect of Cadmium Treatment on the Kidney of Rats: Biochemical and Ultrastructural Studies. Pakistan Journal of Biological Sciences, 10: 3497-3506. 2007.
[11] ChandwanI A and Shuter J Lopinavir/ritonavir in the treatment of HIV-1 infection: a reviewTherapeutics and Clinical Risk Management, 4(5) 1023-1033, 2008.

[12] Reyskens KMSE, T. L. Fisher, J. C. Schisler, O’Connor WG, Rogers AB. Maladaptive Effects of HIV Protease Inhibitors (Lopinavir/ Ritonavir), PLoS ONE. 8(9): e73347.2013.

[13] Nathiya. S, and A. Nandhini..Evaluation of antioxidant effect of Salacia oblonga against aluminum chloride induced visceral toxicity in albino rats Int J Basic Clin Pharmacol. 3(2): 315-31, 2014.

[14] Wendel, A. Enzymatic Basis of Detoxification. 1980 Vol. 1, Academic Press, New York, pp: 333.

[15] Aebi, H. Catalase in vitro. Methods Enzymol.105: 121-126.1984.

[16] OlurisheTO, Kwanashie HO, Anuka JA, Muktar HM and SamboJ.S Renal impact of sub acute lamivudine-artesunate treatment in wistar rats. Afr. J. Pharmacol. Ther. 2(2): 48-53, 2013.

[17] Drury R.A.B, Wallington E. A. and Cameron R, Carleton's Histological Techniques. 4th Edn., Oxford University Press, NY. USA, pp: 279-280. 1967.

[18] American Heart Association, Part 10.1: Life-Threatening Electrolyte Abnormalities Circulation. 112: 121-125, 2005.

[19] Marinella MA. Trimethoprim-induced hyperkalemia: an analysis of reported cases. Gerontology, 45. (4). 209-212. 1999.

[20] Pipoli, A. Tartaglia, A. VSaracino A, Pagano V, Elia Conte P, Santantonio T, Angarano G. Fatal persistent hyponatremia in an AIDS patient HAART and Correlated Pathologies. 3; 58-60.

[21] Kim, Y, W. Antimicrobial-induced Electrolyte and Acid-Base Disturbances. Electrolyte \& Blood Pressure 5:111-115, 2007.

[22] Vel zquez H, Perazella MA, Wright FS, Ellison DH: Renal mechanism of trimethoprim-induced hyperkalemia. Ann Intern Med, 119: 296-301. 1993.

[23] Obirikorang C, Osakunor DNM, Ntaadu B, Adarkwa OK. Renal Function in Ghanaian HIV-Infected Patients on Highly Active Antiretroviral Therapy: A Case-Control Study. PLOS ONE 9(6): e99469. 2014.

[24] Noto H, Kaneko Y, Takano,T and Kurokawa, K. Severe hyponatremia and hyperkalemia induced by trimethoprimsulfamethoxazole in patients with Pneumocystis carinii pneumonia. Internal Medicine. 34. (2), 96-99. 1995

[25] Mori H, Kuroda Y, Imamura S, et al: Hyponatremia and/or hyperkalemia in patients treated with the standard dose of trimethoprim-sulfamethoxazole. Intern Med, 42: 665-669. 2003.

[26] Domingo P, Ferrer S, Cruz J, Morla R, Ris J: Trimethoprimsulfamethoxazole-induced renal tubular acidosis in a patient with AIDS. Clin Infect Dis 20:1435-1437, 1995.

[27] Parke D. V and Piotrowski J. Glutathione: Its role in detoxication of reactive oxygen and environmental chemicals. Acta Pol. Toxicol. 4, 1.1996.

[28] Arai M., Imai H., Kou Mur A T., Yoshid A M., Emoto K., Umeda M. et al. Mitochondrial phospholipid hydroperoxide glutathione peroxidase plays a major role in preventing oxidative injury to cells. J. Biol. Chem. 274, 4924. 1999.

[29] Dzobo K, Naik YS. Effect of selenium on cadmium-induced oxidative stress and esterase activity in rat organs. S Afr J Sci.109, 1-8, 2013.

[30] Mozaffari. A, and Rashidi I, Investigation of protective effect of prednisolone in nephropathy induced by co-trimoxazole in rat Pak J Med Sci (Part-II), 23 (6) 847-850. 2007.

[31] Cunha, A.M., Hagemanna CF, Simões RS, Oliveira-Filhob RM, Simões MJ. Effects of lopinavir-ritonavir combined therapy during rat pregnancy. Morphological and biochemical aspects. Eur J. of Obst \& Gyn and Repro Bio, 133 (1): 60-63. 2006. 